\title{
奇次矩形元导数恢复算子的新构造 及其强超收敛性*
}

\author{
朱起定 ${ }^{*}$ 孟令雄 \\ (湖南师范大学数学与计算机科学学院, 长沙 410081)
}

摘要 考虑奇次矩形元导数的强超收玫问题, 为了做导数后处理, 利用投影型 插值, 提出一类新的离散的最小二乘分片恢复技术, 并且证明此类恢复导数具 有强超收敛性。

\section{关键词 SPR 导数恢复算子 投影性插值 强超收敛性}

\section{1 引言}

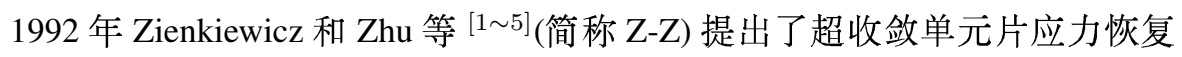
的方案, 即 SPR 技术. 围绕某一点的所有单元称为这一点的单元片. SPR 技术 通过在该单元片上构造具有超收玫特性的光滑应力场来获得节点处的高精度应 力值. 这种光滑应力场一般是通过最小二乘法或离散最小二乘法获得的. 有趣 的是这样一种处理方法还可以得到一些强超收玫结果, 例如对二次元可以在结 点上得到应力的 $O\left(h^{4}\right)$ 阶超收玫性. 在理论上, Zhang 等 ${ }^{[6 \sim 10]}$ 对两点边值问题 以及二维问题的矩形元讨论了这个问题, 并且还证得这一结果对所有偶次元都 是对的, 在结点处导数有 $O\left(h^{k+2}\right)$ 强超收玫性. 但是, 所有这些结果对奇次矩形 元是不成立的.

文献 [11] 利用投影型插值对两点边值问题的有限元方法作了讨论, 获得应 力和位移的一些超收玫结果.

本文讨论了奇次矩形元导数的强超收玫问题, 提出了一类新的离散的最小 二乘分片恢复技术, 利用文献 [12] 提出的投影型插值, 证明了恢复导数具有强 超收玫性, 推广了文献 [11] 的结果.

2004-03-10 收稿, 2004-07-19 收修改稿

* 国家自然科学基金资助项目 (批准号: 10371038)

** E-mail: qdzhu@ @parc2.hunnu.edu.cn 


\section{2 矩形元的投影型插值简介 ${ }^{[12]}$}

考虑二阶椭圆边值问题：设 $u \in H_{0}^{1}(\Omega)$, 使得

$$
\begin{aligned}
& a(u, v)=(f, v), \quad \forall v \in H_{0}^{1}(\Omega), \\
& a(u, v)=\int_{\Omega}\left[\sum_{i j=1}^{2} a_{i j} \partial_{i} u \partial_{j} v+\sum_{j=1}^{2} a_{0 j} \partial_{j} v+a_{00} u v\right] d x d y, \\
& (f, v)=\int_{\Omega} f \cdot v d x d y,
\end{aligned}
$$

其中 $\Omega \subset \mathbb{R}^{2}$ 是有界光滑区域, 系数 $a_{i j}$ 满足一致椭圆条件. 于是由 Lax-Milgram 定理知这个问题存在惟一解.

假定 $\mathcal{T}^{h}$ 是区域 $\Omega \subset \mathbb{R}^{2}$ 上的拟一致的剖分, 在区域内部实现了一致的矩形 剖分. 设

$$
e=\left(x_{e}-h_{e}, x_{e}+h_{e}\right) \times\left(y_{e}-k_{e}, y_{e}+k_{e}\right) \equiv e_{1} \times e_{2}
$$

为任一矩形元. 记 $\Lambda_{\nu}=\{(i, j): 0 \leqslant i \leqslant k, 0 \leqslant j \leqslant k, i+j \leqslant k+\nu\} \cup$ $\{(k, 1),(1, k)\}, \nu=0,1,2, \cdots, k, Q_{k}^{\nu}(e)=\operatorname{span}\left\{x^{i} y^{j}:(i, j) \in \Lambda_{\nu}\right\}$, 若引入记号

$$
k_{i}= \begin{cases}k, & \text { 如果 } 0 \leqslant i \leqslant \max \{1, \nu\} ; \\ k+\nu-i, & \text { 如果 } \max \{1, \nu\}<i<k ; \\ \max \{1, \nu\}, & \text { 如果 } i=k,\end{cases}
$$

则

$$
Q_{k}^{\nu}(e)=\operatorname{span}\left\{x^{i} y^{j}: 0 \leqslant i \leqslant k, 0 \leqslant j \leqslant k_{i}\right\}
$$

我们称

$$
V_{k}^{\nu}(\Omega)=\left\{v \in C(\bar{\Omega}):\left.v\right|_{e} \in Q_{k}^{\nu}(e), \forall e \in \mathcal{T}^{h}\right\}
$$

为 $Q_{k}^{\nu}$ 次有限元空间, 特别在 $\nu=k$ 时为双 $k$ 次有限元空间, 或分别称为 $Q_{k}^{\nu}$ 和 $Q_{k}$ 型有限元空间. 通常称

$$
\begin{array}{ll}
Q_{k}^{0} & \text { 为奇妙族, 或 I0 型 ; } \\
Q_{k}^{1} & \text { 为第 } 1 \text { 中间族, 或 I1 型; } \\
Q_{k}^{\nu}, \nu>1 & \text { 为第 } 2 \text { 中间族, 或 I2 型; } \\
Q_{k}^{k} & \text { 为 } Q_{k} \text { 族. }
\end{array}
$$

为了引入矩形元 $e$ 上的投影型插值, 先讨论一维单元

$$
e_{1}=\left(x_{e}-h_{e}, x_{e}+h_{e}\right)
$$

上的投影型插值. 构作 $L^{2}\left(e_{1}\right)$ 上的规范正交多项式系:

$$
l_{0}(x)=\sqrt{\frac{1}{2}} h_{e}^{-1 / 2},
$$




$$
\begin{aligned}
& l_{i}(x)=\sigma_{i}\left(\frac{d}{d x}\right)^{i}[A(x)]^{i},(i \geqslant 1), \\
& \sigma_{i}=\sqrt{\frac{(2 i+1)}{2}} \frac{1}{i !} h_{e}^{-i-1 / 2}=O\left(h_{e}^{-i-1 / 2}\right), \\
& \ldots
\end{aligned}
$$

其中 $A(x)=\frac{1}{2}\left[\left(x-x_{e}\right)^{2}-h_{e}^{2}\right]$, 并令

$$
\begin{aligned}
\omega_{0}(x)= & h_{e}^{\frac{1}{2}}, \\
\omega_{1}(x)= & \int_{x_{e}-h_{e}}^{x} l_{0}(x) d x \\
& \cdots \\
\omega_{i+1}= & \int_{x_{e}-h_{e}}^{x} l_{i}(x) d x=\sigma_{i}\left(\frac{d}{d x}\right)^{i-1}[A(x)]^{i}, \quad(i \geqslant 1)
\end{aligned}
$$

对 $u \in H^{1}\left(e_{1}\right)$ 定义它的 $k$ 阶投影型插值为

$$
i_{k} u(x)=\sum_{j=0}^{k} \beta_{j} \omega_{j}(x), \quad \forall x \in e,
$$

其中

$$
\left\{\begin{array}{l}
\beta_{0}=h_{e}^{-\frac{1}{2}} u\left(x_{e}-h_{e}\right), \\
\beta_{1}=h_{e}^{-\frac{1}{2}} \sqrt{\frac{1}{2}}\left(u\left(x_{e}+h_{e}\right)-u\left(x_{e}-h_{e}\right)\right), \\
\beta_{j}=\alpha_{j-1}=\left(u^{\prime}, l_{j-1}\right)_{e_{1}}, j \geqslant 2 .
\end{array}\right.
$$

考虑矩形元 $e=e_{1} \times e_{2}$ 上的投影型插值的构作, 显然

$$
\left\{l_{i}(x) \bar{l}_{j}(y)\right\}
$$

是 $L^{2}(e)$ 空间的完备规范正交多项式系，其中 $\bar{l}_{j}(y)$ 是对应于区间 $e_{2}=\left(y_{e}-\right.$ $\left.k_{e}, y_{e}+k_{e}\right)$ 上的规范正交多项式系. 显然, 对于满足 $\partial_{1} \partial_{2} u \in L^{2}(e)$ 的函数 $u$, 也 有展开式

$$
\begin{aligned}
& \partial_{1} \partial_{2} u=\sum_{i=0}^{\infty} \sum_{j=0}^{\infty} \alpha_{i j} l_{i}(x) \bar{l}_{j}(y) \\
& \alpha_{i j}=\left(\partial_{1} \partial_{2} u, l_{i} \bar{l}_{j}\right)_{e}=\int_{e} \partial_{1} \partial_{2} u l_{i}(x) \bar{l}_{j}(y) d x d y .
\end{aligned}
$$

类似于一维情形, 利用 Parseval 等式, 可得

$$
\begin{aligned}
& u(x, y)-u\left(x_{e}-h_{e}, y\right)-u\left(x, y_{e}-k_{e}\right)+u\left(x_{e}-h_{e}, y_{e}-k_{e}\right) \\
= & \sum_{i=0}^{\infty} \sum_{j=0}^{\infty} \alpha_{i j} \omega_{i+1}(x) \bar{\omega}_{j+1}(y)
\end{aligned}
$$

其中 $\bar{\omega}_{j+1}(y)=\int_{y_{e}-k_{e}}^{y} \bar{l}_{j}(y) d y$. 
对于 $u\left(x, y_{e}-k_{e}\right)$, 作为 $x$ 的函数, 有

$$
u\left(x, y_{e}-k_{e}\right)=\sum_{j=1}^{\infty} \beta_{j 0} \omega_{j}(x) \bar{\omega}_{0}(y)+u\left(x_{e}-h_{e}, y_{e}-k_{e}\right),
$$

其中

$$
\beta_{j 0}=k_{e}^{-\frac{1}{2}}\left(\partial_{1} u, l_{j-1}\right)_{e_{1}}=k_{e}^{-\frac{1}{2}} \int_{e^{1}} \partial_{1} u\left(x, y_{e}-k_{e}\right) l_{j-1}(x) d x, j \geqslant 1 .
$$

类似地有

$$
u\left(x_{e}-h_{e}, y\right)=\sum_{j=1}^{\infty} \beta_{0 j} \omega_{0} \bar{\omega}_{j}(y)+u\left(x_{e}-h_{e}, y_{e}-k_{e}\right),
$$

其中

$$
\beta_{0 j}=h_{e}^{-\frac{1}{2}}\left(\partial_{2} u, \bar{l}_{j-1}\right)_{e_{2}}=h_{e}^{-\frac{1}{2}} \int_{e_{2}} \partial_{2} u\left(x_{e}-h_{e}, y\right) \bar{l}_{j-1}(x) d y, j \geqslant 1 .
$$

代入 (2.4) 式得

$$
u(x, y)=\sum_{i=0}^{\infty} \sum_{j=0}^{\infty} \beta_{i j} \omega_{i}(x) \bar{\omega}_{j}(y)
$$

其中 $\beta_{i j}=\alpha_{i-1, j-1}$ (当 $i, j \geqslant 1$ 时). $\beta_{00}=u\left(x_{e}-h_{e}, y_{e}-k_{e}\right) h_{e}^{-\frac{1}{2}} k_{e}^{-\frac{1}{2}}, \omega_{0}(x)=$ $h_{e}^{\frac{1}{2}}, \omega_{0}(y)=k_{e}^{\frac{1}{2}}$.

对于 $k \geqslant 1$, 当 $(x, y) \in e, \forall e \in \mathcal{T}^{h}$ 时, 定义 $u$ 的双 $k$ 次投影型插值为

$$
i_{k} u=\sum_{i=0}^{k} \sum_{j=0}^{k} \beta_{i j} \omega_{i}(x) \bar{\omega}_{j}(y) .
$$

还可以定义所谓 $u$ 的缺损有限元投影型插值

$$
\Pi_{k}^{\nu} u=\sum_{(i, j) \in \Lambda_{\nu}} \beta_{i j} \omega_{i} \bar{\omega}_{j}=\sum_{i=0}^{k} \sum_{j=0}^{k_{i}} \beta_{i j} \omega_{i} \bar{\omega}_{j}, \quad \nu=0,1,2, \cdots, k .
$$

\section{性质 1}

(1) $\Pi_{k}^{\nu} u \in V_{k}^{\nu}(\Omega), \forall u \in H^{2}(\Omega)$;

(2) $\Pi_{k}^{\nu} u\left(x_{e} \pm h_{e}, y_{e} \pm k_{e}\right)=u\left(x_{e} \pm h_{e}, y_{e} \pm k_{e}\right)$;

(3) 在单元 $e$ 的各边上有

$$
\begin{aligned}
& \Pi_{k}^{\nu} u\left(x_{e}-h_{e}, y\right)=\Pi_{*, k} u\left(x_{e}-h_{e}, y\right), \\
& \Pi_{k}^{\nu} u\left(x, y_{e}-k_{e}\right)=\Pi_{k, *} u\left(x, y_{e}-k_{e}\right),
\end{aligned}
$$

其中 $\Pi_{k, *}, \Pi_{k, *}$ 分别表示在 $y, x$ 固定时的一维 $k$ 投影型插值算子，从而表明 $\Pi_{k}^{\nu}$ 是从 $H^{2}(\Omega)$ 到 $V_{k}^{\nu}$ 型有限元空间

$$
V_{k}^{\nu}(\Omega)=\operatorname{span}\left\{v \in C(\Omega):\left.v\right|_{e} \in Q_{k}^{\nu}(e)\right\}
$$

的一个插值算子.

(4) $\left|u-\Pi_{k}^{\nu} u\right|_{m, q, e} \leqslant C h_{e}^{k+1-m}|u|_{k+1, q, e}(1 \leqslant q \leqslant \infty, m=0,1,2)$.

注 1 性质 1(3) 确保了插值 $\Pi_{k}^{\nu} u$ 在整个区域 $\Omega$ 上的连续性. 
由定义显然还有如下正交结果:

推论 1 对任意矩形单元 $e$ 有

(1) $\left(\partial_{i}\left(u-\Pi_{k}^{\nu} u\right), p\right)_{e}=0, \forall p \in P_{k-1}(e), \quad i=1,2(\nu \geqslant 1)$;

(2) $\left(u-\Pi_{k}^{\nu} u, p\right)_{e}=0, \forall p \in P_{k-2}(e) \quad(\nu \geqslant 2)$.

利用投影型插值的性质及文献 $[13,14]$ 的离散 Green 函数理论, 内超收玫估计 理论， Lin 和 Zhu 证得如下超逼近结果 (参见文献 [12] §1.4.3 命题 5 及定理 3.4.2):

定理 1 假定 $D \subset \subset \Omega$ 被拟一致矩形剖分覆盖, $E \subset \subset D$, 设 $u^{h}, \Pi_{k}^{\nu} u \in$ $V_{k}^{\nu}(\Omega)$ 分别是边值问题 (2.1) 的有限元逼近和投影型插值，那么有超逼近：对 $\nu \geqslant 2$ (对数因子 $|\log h|$ 忽略不记),

$$
\left\|u^{h}-\Pi_{k}^{\nu} u\right\|_{m, \infty, E} \leqslant C h^{k+2-m}|u|_{k+3, \infty, D}+\left\|u-u^{h}\right\|_{-s, \Omega}(k \geqslant 2-m, m=0,1),
$$

当 $a_{11}=a_{22}=1, a_{12}=a_{21}=0, a_{0 j}=0$ 时, 若 $D$ 被一致网格覆盖, 则还有强超 逼近: 对 $\nu \geqslant 2$,

$$
\left\|u^{h}-\Pi_{k}^{\nu} u\right\|_{m, \infty, E} \leqslant C h^{k+3-m}|u|_{k+3, \infty, D}+\left\|u-u^{h}\right\|_{-s}(k \geqslant 3-m, m=0,1),
$$

其中 $s$ 是任意固定的非负正数.

\section{3 奇次矩形元导数恢复算子的新构造}

现在设 $E$ 是任意内结点 $z_{0}=\left(x_{0}, y_{0}\right)$ 处的单元片, 它由围绕 $z_{0}$ 的 4 个矩形 单元之并构成 (见图 1), 并构作线单元片

$$
E_{1}=\left\{(x, y) \in E: y=y_{0}\right\}, \quad E_{2}=\left\{(x, y) \in E: x=x_{0}\right\} .
$$

对于偶次矩形元, Z-Z 是采用单元片 $E$ 中 4 个矩形单元 $e^{(i)}$ 的 $k$ 阶 Gauss 分 点集

$$
G=\bigcup_{i=1,2,3,4}\left\{(x, y) \in e^{(i)}: l_{k}(x)=0, \bar{l}_{k}(y)=0\right\}
$$

为样本点构作的, 这种构作方法对奇次元只能得到 $O\left(h^{k+1}\right)$ 的普通超收玫结果, 不能获得强超收玫性.

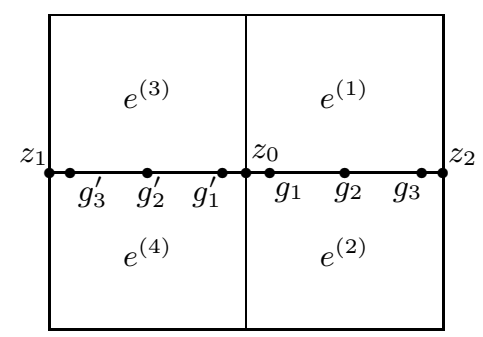

图 1

www.scichina.com 
对于奇次矩形元, 我们分别对线单元片 $E_{1}$ 和 $E_{2}$ 采用样本点集

$$
\begin{aligned}
& G_{1}=\cup_{i=1,3}\left\{\left(x, y_{0}\right) \in \bar{e}^{(i)}: \alpha_{k}^{1} l_{k}(x)+\alpha_{k+1}^{1} l_{k+1}(x)=0\right\}, \\
& G_{2}=\cup_{i=1,2}\left\{\left(x_{0}, y\right) \in \bar{e}^{(i)}: \alpha_{k}^{2} \bar{l}_{k}(y)+\alpha_{k+1}^{2} \bar{l}_{k+1}(y)=0\right\},
\end{aligned}
$$

其中 $\alpha_{m}^{1}=\int_{e_{1}}\left(x-x_{0}\right)^{k+1} l_{m}(x) d x, \alpha_{m}^{2}=\int_{e_{2}}\left(y-y_{0}\right)^{k+1} \bar{l}_{m}(y) d y(m=k . k+1)^{1)}$, 来构作 $x$ 方向和 $y$ 方向的导数恢复算子. 任给 $w \in V_{k}^{\nu}(E)$, 在样本集 $G_{i}(i=1,2)$ 上构作极小问题: 设 $v_{i} \in P_{k+1}\left(E_{i}\right)$, 使得

$$
\left\|\left|v_{i}-\partial_{i} w \|\right|_{i}=\min \left\{\left\|\left|v-\partial_{i} w \|\right|_{i}: v \in P_{k+1}\left(E_{i}\right)\right\},\right.\right.
$$

其中 $\|v\| \|_{i}^{2}=\sum_{g \in G_{i}}[v(g)]^{2}$. 由于 $k>1$ 为奇数, $2 k \geqslant k+3$, 这个最小二乘法问 题存在惟一解, 故可以定义算子

$$
R_{i}: w \in V_{k}^{\nu}(E) \rightarrow v_{i} \in P_{k+1}\left(E_{i}\right) .
$$

容易证明如下性质:

r1) 若 $v \in P_{k+2}\left(E_{i}\right)$, 则

$$
\left.v\right|_{E_{i}} \equiv 0 \Longleftrightarrow v(g)=0, \quad \forall g \in G_{i} \Longleftrightarrow\|\mid v\|_{i}=0,
$$

且 $R_{i} v=\partial_{i} v$;

$\mathrm{r} 2$ ) 若令 $\langle v, w\rangle_{i}=\sum_{g \in G_{i}} v(g) w(g)$, 则问题 (3.2) 等价于方程

$$
\left\langle\partial_{i} w-R_{i} w, v\right\rangle_{i}=0, \quad \forall v \in P_{k+1}\left(E_{i}\right),
$$

其中 $R_{i} w=v_{i}$;

r3) $\left\|\left|R_{i} w\left\|\left.\right|_{i} \leqslant\right\| \partial_{i} w \|_{i} ;\right.\right.$

$\mathrm{r} 4)$ 若 $w$ 关于点 $z_{0}$ 偶对称，则 $R_{i} w$ 关于点 $z_{0}$ 奇对称，从而 $R_{i} w\left(z_{0}\right)=0$.

注 2 事实上, $\mathrm{r} 1)$ 是因为 $G_{i}$ 具有 $2 k(\geqslant k+3)$ 个不同的样本点. 二次泛函 的极值原理蕴含 $\mathrm{r} 2$ ) 和 $\mathrm{r} 3$ ).

我们只需详细证明 $\mathrm{r} 4$ ). 不妨设 $z_{0}$ 是原点. 如果 $w$ 是偶对称的, 那么 $\partial_{i} w$ 是 奇对称的. 故对于 $v=R_{i} w$, 有

$$
\begin{aligned}
\left\|\left|v-\partial_{i} w \|\right|_{i}^{2}\right. & =\sum_{g \in G_{i}}\left|v(g)-\partial_{i} w(g)\right|^{2}=\sum_{g \in G_{i}}\left|(-v(-g))-\left(-\partial_{i} w(-g)\right)\right|^{2} \\
& =\sum_{g \in G_{i}}\left|\bar{v}(g)-\partial_{i} w(g)\right|^{2}=\left\|\left|\bar{v}-\partial_{i} w \|\right|_{i}^{2},\right.
\end{aligned}
$$

其中 $\bar{v}(x)=-v(-x)$, 而第 2 个等式由 $G_{i}$ 的对称性得到，因此 $\bar{v}$ 和 $v$ 都是极小问 题 (3.2) 的解. 解的惟一性导出 $\bar{v}=v$, 即 $R_{i} w$ 是奇对称的.

引理 1 当 $k>1$ 为奇数时, 算子 $R_{i}$ 满足如下两条件:

A) $\left\|R_{i} w\right\|_{0, \infty, E_{i}} \leqslant C\left\|\partial_{i} w\right\|_{0, \infty, E_{i}}, \forall w \in V_{k}^{\nu}(E)$;

B) $\partial_{i} u\left(z_{0}\right)-R_{i} u^{I}\left(z_{0}\right)=0, \forall u \in P_{k+2}\left(E_{i}\right)$,

此处 $u^{I}=i_{k} u$ 是二维插值 $\Pi_{k}^{\nu} u$ 在线段 $E_{i}$ 上的限制, 实际上是一维的 $k$ 阶投影 型插值 (见性质 1(3)).

1) 注意这里 $e_{1}, e_{2}$ 是矩形元 $e$ 的两条边, $G_{i} \subset E_{i}$, 关于中心 $z_{0}$ 对称而且正好是 $2 k$ 个点, 详见附录 
证 由 $\mathrm{r} 3$ ) 并注意 $G_{i}$ 的元素的个数为 $2 k$, 得

$\left\|R_{i} w\right\|_{0, \infty, E_{i}} \leqslant C\left\|\left|R_{i} w\left\|\left|\leqslant C\left\|\partial_{i} w\right\|\right| \leqslant C \sqrt{2 k}\right\| \partial_{i} w \|_{0, \infty E_{i}}\right.\right.$,

A) 证毕. 证 B), 不妨设 $i=1$, 当 $u \in P_{k}\left(E_{1}\right)$ 时, 有 $u^{I}=u$, 结论成立, 故只需对 $u=u\left(x, y_{0}\right)=\left(x-x_{0}\right)^{m}(m=k+1, k+2)$ 验证 $\partial_{1} u\left(z_{0}\right)-R_{1} u^{I}\left(z_{0}\right)=0$ 就可以 了. 注意 $k+1$ 是偶数和性质 $\mathrm{r} 4$ ), 只需验证 $m=k+2$ 的情形. 由一维投影型插 值定义 (2.2) 式和样本点集 $G_{1}$, 得

$$
\partial_{1} u\left(x, y_{0}\right)=\partial_{1} i_{k} u\left(x, y_{0}\right)+m \alpha_{k}^{1} l_{k}(x)+m \alpha_{k+1}^{1} l_{k+1}(x)=\partial_{1} u^{I}\left(x, y_{0}\right), \forall x \in G_{1},
$$

于是由算子 $R_{1}$ 的定义得 $R_{1} u=R_{1} u^{I}$, 最后注意 $\partial_{1} u \in P_{k+1}\left(E_{1}\right)$ 就得到 $\partial_{1} u=$ $R_{1} u=R_{1} u^{I}$, 于是 $\mathrm{B}$ ) 得证从而引理证毕.

\section{4 强超收敛定理及其证明}

综上所述, 我们有如下重要定理:

定理 2 设 $k>1$ 为奇数, $u^{h} \in V_{k}^{\nu}$ 是 $u$ 的有限元逼近, 导数恢复算子 $R_{i}: V_{k}^{\nu}\left(E_{i}\right) \rightarrow P_{k+1}\left(E_{i}\right)(i=1,2)$ 满足条件 $\left.\mathrm{A}\right)$ 和 $\left.\mathrm{B}\right)$, 则当 $E \subset \subset D$ 且 $D \subset \subset \Omega$ 被 拟一致矩形网格覆盖, 且在 $\nu \geqslant 2$ 时, 有超收玫估计

$$
\left|\partial_{i} u\left(z_{0}\right)-R_{i} u^{h}\left(z_{0}\right)\right| \leqslant C h^{k+1}|u|_{k+2, \infty, D}+\left\|u-u^{h}\right\|_{-s, \Omega} .
$$

进一步若 $D \subset \subset \Omega$ 被一致矩形网格覆盖, 且当 $\nu \geqslant 2$ 时, 有导数的强超收玫估计

$$
\left|\partial_{i} u\left(z_{0}\right)-R_{i} u^{h}\left(z_{0}\right)\right| \leqslant C h^{k+2}|u|_{k+3, \infty, D}+\left\|u-u^{h}\right\|_{-s, \Omega}
$$

证 只需证明后一个较难的结果就可以了. 由条件 B) 并利用 Bramble-Hilbert 引理得 $\left|\partial_{i} u\left(z_{0}\right)-R_{i} u^{I}\left(z_{0}\right)\right| \leqslant C h^{k+2}|u|_{k+3, \infty, E_{i}}$; 又由条件 A) 以及 (2.8) 式得

$$
\left|R_{i}\left(u^{I}-u^{h}\right)\left(z_{0}\right)\right| \leqslant C\left\|\partial_{i}\left(u^{I}-u^{h}\right)\right\|_{0, \infty, E_{i}} \leqslant C h^{k+2}\|u\|_{k+3, \infty, D}+\left\|u-u^{h}\right\|_{-s},
$$

于是借助于三角不等式得证结果. 这里 $u^{I}=\left.\Pi_{k}^{\nu} u\right|_{E_{i}}$.

附注 满足定理条件 A) 和 B) 的算子 $R_{i}$ 并不仅仅限于前面构作的算子. 我 们也可以在 $G_{i}$ 中选取 $k+3$ 个关于 $z_{0}$ 点对称的点 $G_{i}^{\prime}=\left\{g_{ \pm 1}, g_{ \pm 2} \cdots, g_{ \pm s}\right\}(s=$ $(k+3) / 2)$, 以 $G_{i}^{\prime}$ 为结点构作 $k+2$ 次插值算子 $I_{h}$, 则算子 $R_{i}:=I_{h} \partial_{i}$ 也满足条件 A) 和 B).

\section{5 数例分析}

本节用实例来说明用这种方法所获得的高精度和强超收玫率. 仍然考虑模 型问题

$$
\begin{aligned}
& -\Delta u=f, \text { 在 } \Omega=[0,1] \times[0,1] \text { 中, } \\
& \left.u\right|_{\partial \Omega}=0 .
\end{aligned}
$$

这里假设 $f$ 是对应于真解 $u=10 x \sin \pi x \sin \pi y$ 的函数. 假设在 $\Omega$ 上实现了一致 剖分, 并且运用双三次矩形元解问题 (5.1), 考察在点 $x_{0}=(0.25,0.25)$ 的情形 (见 表 1). 
表 1 双三次元在点 $z_{0}=(0.25,0.25)$ 处沿 $x$ 方向的导数误差表

\begin{tabular}{ccc}
\hline$h$ & $\left|\sigma-\sigma^{\star}\right|$ & $\left|\sigma-\sigma^{R}\right|$ \\
\hline$\frac{1}{4}$ & $4.137179297103444(-5)$ & $3.875638802971793(-2)$ \\
$\frac{1}{8}$ & $6.474424836255821(-7)$ & $2.481111253091581(-3)$ \\
$\frac{1}{12}$ & $5.575402539648167(-8)$ & $4.922390230728269(-4)$ \\
$\frac{1}{16}$ & $9.788458044113213(-9)$ & $1.559844826211077(-4)$ \\
收敛速度 & 6 & 4 \\
\hline
\end{tabular}

真解沿 $x$ 方向的导数 $\sigma=8.92699081698724 . \sigma^{R}$ 表示用 SPR 产生的恢复导数; $\sigma^{\star}=R_{1} u^{h}\left(z_{0}\right)$ 表示用我们的方法产生的恢复导数

我们可以看出, 恢复值 $\sigma_{x}^{\star}$ 有强超收玫性, 而且它的收玫阶为 $O\left(h^{6}\right)$. 这是一 个令人惊讶和振奋的结果.

\section{参考文献}

1 Zienkiewicz O C, Zhu J Z. The superconvergence patch recovery and a posteriori estimates. Part 1: The recovery technique. Int J Numer Methods Engrg, 1992, 33: 1331 1364

2 Zienkiewicz O C, Zhu J Z. The superconvergence patch recovery and a posteriori estimates. Part 2: Error estimates and adaptivity. Int J Numer Methods Engrg, 1992, 33: 1365 1382

3 Zienkiewicz O C, Zhu J Z. The superconvergence patch recovery (SPR) and adaptive finite element refinement. Comput Methods Appl Mech Engrg, 1992, 101: 207 224

4 Zienkiewicz O C, Zhu J Z. Superconvergence and the superconvergent patch recovery finite element. Anal Design, 1995, 19: 11 23

5 Zienkiewiz O C, Zhu J Z, Wu J. Superconvergence patch recovery techniques— - some further tests. Commun Num Math Meth Erg, 1993, 9: 251 258

6 Zhang Z M. Ultraconvergence of the patch recovery technique. Math Comp, 1996, 65(216): 1431 1437

7 Zhang Z M. Ultraconvergence of the patch recovery technique II. Math Comp, 2000, 69: 141 158

8 Zhang Z M, Zhu J Z. Analysis of the superconvergence patch recovery techniques and a posteriori error estimator in the finite element method (I). Comput Methods Appl Mech Engrg, 1995, 123: 173 187

9 Zhang Z M, Zhu J Z. Analysis of the supencorvergent patch recovery technique and a posteriori error estimator in the finite element method (II). Comput Methods Appl Mech Engrg, 1998, 163: 159 170

10 Zhang Z M, H D Victorg Jr. Mathematical analysis of Zienkiewicz-Zhu's derivative patch recovery technique. Numer Methods for PDEs, 1996, 12: 507 524

11 Zhu Q D, Zhao Q H. SPR technique and finite element correction. Numer Math, 2003, 96: 185 196

12 林群, 朱起定. 有限元的预处理和后处理理论. 上海: 上海科技出版社, 1994. 7 109

13 Zhu Q D. Natural inner superconvergence for the finite element method. In: Feng K, Lions J L, eds. Proceedings of the China-France Symposium on Finite Element Methods. Beijing: Science Press, Gorden and Breach, 1983. 935 960

14 朱起定, 林群. 有限元超收玫理论. 长沙: 湖南科技出版社, 1989. 92 135 


\section{附录 样本点的选取}

命题 方程

$$
\alpha_{k} L_{k}(x)+\alpha_{k+1} L_{k+1}=0
$$

在 $[-1,1]$ 内有 $k$ 个不同的根 $\xi_{1}, \xi_{2}, \cdots, \xi_{k}$, 其中 $\left\{L_{k}(x)\right\}$ 为 $[-1,1]$ 上的规范正交多项式 (Legendre 多项式), 即

$$
L_{k}(t)=\sqrt{\frac{2 k+1}{2}} \frac{1}{k !} \frac{d^{k}}{d x^{k}}\left(\frac{1}{2}\left(x^{2}-1\right)\right)^{k}
$$

和

$$
\alpha_{i}=(k+2) \int_{-1}^{1}(1+x)^{k+1} L_{i}(x) d x, i=k, k+1 .
$$

证 令 $F(x)=\alpha_{k} L_{k}(x)+\alpha_{k+1} L_{k+1}(x)$, 并注意多项式 $L_{k}$ 在 $(-1,1)$ 之中有 $k$ 个单根

$$
-1<h_{1}<h_{2}<\cdots<h_{k}<1,
$$

而且在通过这些根时符号交错变化， $L_{k+1}$ 的根 $h_{j}^{\prime}$ 也具有同样性质，且满足

$$
-1<h_{1}^{\prime}<h_{1}<h_{2}^{\prime}<h_{2}<\cdots<h_{k}^{\prime}<h_{k}<h_{k+1}^{\prime}<1,
$$

因为 $F\left(h_{j}^{\prime}\right)=\alpha_{k} L_{k}\left(h_{j}^{\prime}\right)(j=1,2, \cdots, k+1)$ 的符号也是交替出现的, 由介值定理立即得 $F(x)$ 在 $(-1,1)$ 之中有 $k$ 个不同的根.

前面指出, 对于线单元片 $D_{1}=\left[x_{0}-h, x_{0}\right] \cup\left[x_{0}, x_{0}+h\right]$, 如果取

$$
G=\left\{x_{0} \pm\left(1+\xi_{i}\right) h: i=1,2, \cdots, k\right\}
$$

为样本点, 在 $D_{1}$ 上对导数进行 $k+1$ 次曲线拟合, 就可以在 $x_{0}$ 处得到强超收玫性.

设 $D=[-2,2]$ 为参考线单元片, 将 $D$ 上各种奇次元所应取的样本点列表如下:

$$
\begin{aligned}
& k=3 \pm 0.2418824049, \pm 1.0353424099, \pm 1.7871032702 ; \\
& k=5 \pm 0.0968152457, \pm 0.4729063288 ; \\
& \pm 1.0150951112, \pm 1.5486221515, \pm 1.9086504188 ; \\
& k=7 \quad \pm 0.0512427245, \pm 0.2600493426, \pm 0.5970566257, \\
& \pm 1.0034302824, \pm 1.4086751287, \pm 1.7430409659, \\
& \pm 1.9494385811 .
\end{aligned}
$$

